EPJ Web of Conferences 101, 06056 (2015)

DOI: $10.1051 /$ epjconf/ 201510106056

(C) Owned by the authors, published by EDP Sciences, 2015

\title{
Transiting Sub-stellar companions of Intermediate-mass stars
}

\author{
Daniel Sebastian ${ }^{1, a}$, Eike W. Guenther ${ }^{1}$, Ulrich Heber $^{2}$, Stephan Geier $^{3}$, Sascha Grziwa ${ }^{4}$, and the \\ CoRoT-team
}

1 Thueringer Landessternwarte Tautenburg, Sternwarte 507778 Tautenburg, Germany

2 Dr. Karl Remeis Observatory \& ECAP, University Erlangen-Nürnberg, Germany

3 European Southern Observatory (ESO), Karl-Schwarzchild Str. 2 D-85748 Garching bei München, Germany

${ }^{4}$ Rheinisches Institut für Umweltforschung, Abteilung Planetenforschung, an der Universität zu Köln

\begin{abstract}
We use the CoRoT-survey to search for transiting close-in planets of intermediate-mass stars $\left(M_{*}=1.3-2.1 M_{\odot}\right)$. We present recent results of our survey. RVsurveys and direct imaging campaigns showed, that intermediate-mass main-sequence stars have more massive planets then solar-like stars. Even brown dwarfs have been found. In our study we concentrated on short-period planets for which a mass-determination is possible. The detection of close-in planets of intermediate-mass stars put strong constraints on the timescales of the formation and migration. We already have identified transiting Jupiter-like planet candidates with short orbital periods and observed these candidates with high-resolution echelle-spectrographs at various Telescopes.
\end{abstract}

\section{Introduction}

It is known that planets form within a few million years in the circumstellar disk of young stars. It is also expected that the properties of the planets are closely related to the properties of the disks. Studies of dusty disks of young stars with the Spitzer satellite have shown that the mass of the disk increases with the mass of the star, but its lifetime decreases ([9]). To understand the formation of planets it is crucial to learn how migration in such a disc works. Up to now we do not know much about closein planets of intermediate mass stars (IMSs). However, one disadvantage of IMSs is that they rotate rapidly as long as they are on the main-sequence. Also stellar oscillations induce line profile variations that depend on time and pulsation type. These effects decrease the accuracy of the radial velocity (RV) measurements, one of the most important tools to characterise the mass of the planets. Once these stars evolve to giant stars, the rotation velocity decreases. that makes it easier to analyse them. RVSurveys of giant stars and direct imaging surveys have shown that the frequency of massive planets orbiting at distances larger than 0.5 AU, is twice as high for IMSs than for solar type stars ([6,7]; [12]). Depending on the speed of migration theoretical expectations ([8];[1])) agree with these results and predict that the frequency of close-in planets could be low. By analysing the frequency of close-in, massive planets of stars more massive than the Sun we directly determine the rate of migration. To carry out a dedicated survey to find these planets it is essential that the survey is particularly sensitive for short-period planets and that stars of different masses are surveyed. The CoRoT survey fulfills these criteria and in here we will present our survey for transiting, close-in planets of IMSs.

\footnotetext{
a e-mail: sebastian@tls-tautenburg.de
}

This is an Open Access article distributed under the terms of the Creative Commons Attribution License 4.0, which permits unrestricted use, distribution, and reproduction in any medium, provided the original work is properly cited. 


\section{RV-survey}

As input list for our survey we identified IMSs in the CoRoT fields by using spectral types derived from low resolution spectroscopy ([10];[3]) and photometric observations provided in the EXODAT database ([2]). To analyse the CoRoT light curves of these stars for transits we used the algorithms in Exotrans ([5]). We found 19 promising candidates that have been followed up to exclude false positives. We obtained low-resolution spectra with the TWIN at $3.5 \mathrm{~m}$ at Calar Alto and the Nasmyth spectrograph at the $2.0 \mathrm{~m}$ TLS observatory of all candidates for which only photometric spectral classification was available. Spectral types were obtained by comparing the spectra to a library of stellar spectra ([11]). Three candidates with photometric spectral types of IMSs were found to be solar type stars and are not further analysed.

For radial velocity determinations well stabilised high-resolution echelle spectrographs are absolutely essential. To rule out binaries we use the Sandiford at the $2.1 \mathrm{~m}$ telescope at McDonald Observatory. The well stabilised spectrographs CAFE at $2.2 \mathrm{~m}$ at Calar Alto, UVES at VLT as well as FIES at NOT are used to analyse high priority targets in detail. Since many of the intermediate-mass stars rotate rapidly $(>50 \mathrm{~km} / \mathrm{s})$ the cross correlation method often gives no correlation. Especially for spectra with low $\mathrm{S} / \mathrm{N}$. Therefore the spectra are analysed using a least squares fit method to an model spectrum. An algorithm was developed that matches the model spectrum to all orders of the observed echelle spectrum simultaneously. The echelle- blaze function is taken into account for weighting the fit. To increase accuracy all observed spectra are velocity corrected and combined to obtain a high $\mathrm{S} / \mathrm{N}$ spectrum of the target that is used as template. With this method we can achive an accuracy of $\sim 200 \mathrm{~m} / \mathrm{s}$ for fast rotating stars $(v \operatorname{sini} \sim 90 \mathrm{~km} / \mathrm{s}$ ). Some of our candidates are pulsating stars. Pulsations induce strong line profile variations that leads to deviations from the center-of-mass velocity by using the CCF or least squares fit-methods. To overcome these variations we analyse the line profile and calculate the integrated center of the line.

\section{Results and Conclusions}

For 16 candidates we obtained high resolution echelle spectra. Up to now, more than 180 spectra were analysed. The preliminary identification is summarised in the following list:

- 8 objects turned out to be binaries

- 5 objects are candidates for brown dwarfs.

- 1 object turned out to be a planet candidate ([4])

- 2 objects are currently unsolved and will be observed further.

So far we did not detect any close-in planet around stars $M_{*}>1.5 M_{\odot}$, although our measurements are precise enough to detect them. Four of the brown dwarf candidates are in the border region between brown dwarfs and low mass stars! If they are real we can conclude that the frequency of brown dwarfs is increasing with stellar mass.

\section{References}

1. Alibert, Y. et al., A\&A 526, A63 (2011)

2. Deleuil, M., Meunier, J.C., Moutou, C., et al., AJ 138, 649 (2009)

3. Guenther, E. W., Gandolfi, D., Sebastian, D., et al., A\&A 543, A125 (2012)

4. Guenther, E.W., Cusano, F., Deeg, H., et al., this proceedings

5. Grziwa, S., Pätzold, M., \& Carone, L., MNRAS 420, 1045 (2012)

6. Johnson, J.A., Howard, A.W., Bowler, B.P., et al., PASP 122, 701 (2010)

7. Johnson, J.A., Bowler, B.P., Howard, A.W., et al., ApJL 721, L153 (2010)

8. Kennedy, G.M., \& Kenyon, S.J., ApJ 673, 502 (2008)

9. Mamajek, E.E., American Institute of Physics Conference Series 1158, 3 (2009)

10. Sebastian, D., Guenther, E. W., Schaffenroth, V., et al., A\&A 541, A34 (2012)

11. Valdes, F., Gupta, R., Rose, J. A., Singh, H. P., \& Bell, D. J., ApJS 152, 251 (2004)

12. Vigan, A. et al., A\&A 544, 9 (2012) 